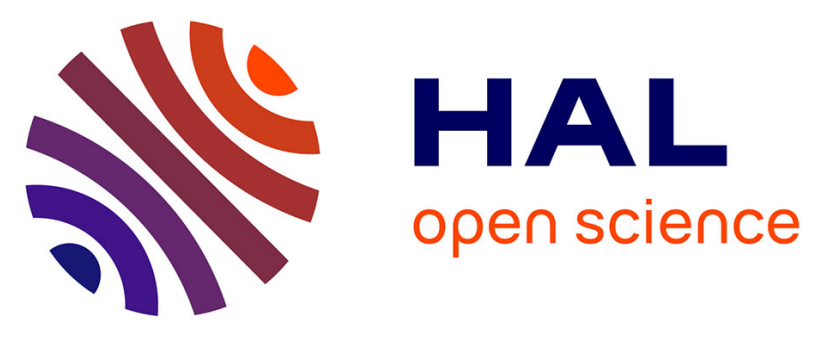

\title{
Additive manufacturing process creates local surface roughness modifications leading to variation in cell adhesion on multifaceted TiAl6V4 samples
}

Augustin Lerebours, Pascale Vigneron, Salima Bouvier, Alain Rassineux, Maxence Bigerelle, Christophe Egles

\section{To cite this version:}

Augustin Lerebours, Pascale Vigneron, Salima Bouvier, Alain Rassineux, Maxence Bigerelle, et al.. Additive manufacturing process creates local surface roughness modifications leading to variation in cell adhesion on multifaceted TiAl6V4 samples. International Journal of Bioprinting, 2019, 16, pp.e00054. 10.1016/j.bprint.2019.e00054 . hal-02407732

\section{HAL Id: hal-02407732 \\ https://hal.utc.fr/hal-02407732}

Submitted on 21 Dec 2021

HAL is a multi-disciplinary open access archive for the deposit and dissemination of scientific research documents, whether they are published or not. The documents may come from teaching and research institutions in France or abroad, or from public or private research centers.
L'archive ouverte pluridisciplinaire HAL, est destinée au dépôt et à la diffusion de documents scientifiques de niveau recherche, publiés ou non, émanant des établissements d'enseignement et de recherche français ou étrangers, des laboratoires publics ou privés.

\section{(c) (1) $\$$}

Distributed under a Creative Commons Attribution - NonCommercial| 4.0 International 
Title: Low dose Rituximab for Pre-emptive Treatment of Epstein Barr Virus Reactivation after Allogenic Hematopoietic Stem Cell Transplantation

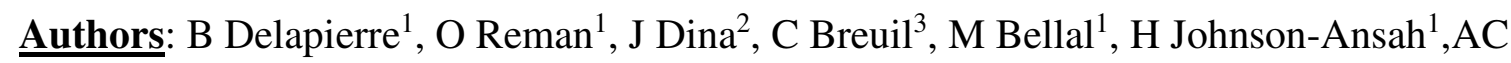
$\mathrm{Gac}^{1}, \mathrm{G}_{\text {Damaj }}{ }^{1}$, S Chantepie ${ }^{1}$

1. Normandie Univ, UNICAEN, CHU de Caen Normandie, 14000 Caen, France

2. CHU de Caen, Department of Virology, 1400 Caen, France; Normandie Univ, France

3. Pharmacie du Centre Hospitalo-universitaire (CHU) de Caen, 14000, Caen, France

Correspondance: Chantepie Sylvain, Institut d'Hématologie de Basse Normandie, Avenue de la côte de Nacre, 14033, Caen, France

chantepie.s@chu-caen.fr

02.31.27.20.73 
Table 1: Comparison between the chemical composition of Ti64 samples-as-SLM and Ti64 powders

\begin{tabular}{|c|c|c|c|c|c|c|c|c|c|c|}
\hline \multicolumn{11}{|c|}{ Composition of Ti64-ELI powders } \\
\hline Al & & C & Fe & $\mathbf{H}$ & $\mathbf{N}$ & $\mathbf{0}$ & $\mathrm{Ti}$ & $\mathbf{v}$ & Others & $\begin{array}{l}\text { All } \\
\text { Others }\end{array}$ \\
\hline 6.2 & & 0.01 & 0.14 & 0.002 & 0.01 & 0.07 & Bal & 4.1 & $<0.1$ & $<0.01$ \\
\hline \multicolumn{11}{|c|}{ Composition of Ti64-ELI samples-as SLM in the surface } \\
\hline Al & & C & Fe & $\mathbf{H}$ & $\mathbf{N}$ & 0 & $\mathrm{Ti}$ & $\mathbf{v}$ & Others & $\begin{array}{l}\text { All } \\
\text { Others }\end{array}$ \\
\hline & $\begin{array}{l}4,97 \pm \\
0,26\end{array}$ & - & $\begin{array}{l}0,19 \pm \\
0,38\end{array}$ & - & - & - & $\begin{array}{l}91,64 \\
\pm 1,26\end{array}$ & $\begin{array}{l}2,74 \pm \\
0,20\end{array}$ & - & - \\
\hline
\end{tabular}


Table 2: Manufacturing parameters and stripes hatching strategy

\begin{tabular}{|c|c|c|c|c|}
\hline & Puissance & Vitesse & Gaz de protection & Argon \\
\hline Bulk filling & $175 \mathrm{~W}$ & $775 \mathrm{~mm} / \mathrm{s}$ & Pattern type & stripes \\
\hline Contour filling & $100 \mathrm{~W}$ & $525 \mathrm{~mm} / \mathrm{s}$ & Hatch vector & $120 \mu \mathrm{m}$ \\
\hline Border & $100 \mathrm{~W}$ & $525 \mathrm{~mm} / \mathrm{s}$ & Distance inter ext. & $60 \mu \mathrm{m}$ \\
\hline & & & Layer width & $30 \mu \mathrm{m}$ \\
\hline \multirow[t]{2}{*}{$\begin{array}{l}\text { Stripes hatching } \\
\text { strategy }\end{array}$} & & & & \\
\hline & & uche $n$ & \multicolumn{2}{|c|}{ Couche $n+1$} \\
\hline
\end{tabular}


Table 3: Measured roughness parameters on the different surfaces of the complex Ti6Al4V

\begin{tabular}{|c|c|c|c|c|c|}
\hline & Zone 1 & Zone 2 & Zone 3 & Zone 4 & $\begin{array}{l}\text { Laser } \\
\text { grooves }\end{array}$ \\
\hline $\begin{array}{l}\text { Number of partially } \\
\text { melted powder } / \mathrm{mm}^{2}\end{array}$ & $188,3 \pm 35.9$ & $412.7 \pm 89.7$ & $500.9 \pm 81.0$ & $\begin{array}{c}1192.1 \pm \\
89.4\end{array}$ & NA \\
\hline Average height: Sa $(\mu \mathrm{m})$ & $8,7 \pm 0.6$ & $9,9 \pm 1.1$ & $10,5 \pm 0.2$ & $19.2 \pm 1.2$ & $0.11 \pm 0.03$ \\
\hline $\begin{array}{c}\text { Maximum height : Sz } \\
(\mu \mathrm{m})\end{array}$ & $125,8 \pm 15.1$ & $138,07 \pm 6.8$ & $161,3 \pm 4.9$ & $230.4 \pm 2.5$ & $0.87 \pm 0.42$ \\
\hline $\begin{array}{l}\text { Root mean square : Sq } \\
\qquad(\mu \mathrm{m})\end{array}$ & $11,8 \pm 0.6$ & $12,8 \pm 1.4$ & $13,8 \pm 0.6$ & $24,1 \pm 1.8$ & $0.13 \pm 0.3$ \\
\hline $\begin{array}{l}\text { core roughness depth: } \\
\qquad \mathbf{S k}(\boldsymbol{\mu m})\end{array}$ & $25,16 \pm 3.7$ & $29,48 \pm 4.6$ & $31,58 \pm 1.6$ & $61.8 \pm 2.9$ & $0.22 \pm 0.3$ \\
\hline $\begin{array}{l}\text { reduced peak height : } \\
\text { SpK }(\mu \mathrm{m})\end{array}$ & $21,0 \pm 0.7$ & $18,2 \pm 1.3$ & $19,7 \pm 2.9$ & $18,2 \pm 1.0$ & $0,1 \pm 0.01$ \\
\hline $\begin{array}{l}\text { reduced valley depth: } \\
\text { SvK }(\mu \mathrm{m})\end{array}$ & $8,2 \pm 1.6$ & $9,1 \pm 0.8$ & $10,2 \pm 0.2$ & $27,4 \pm 5.2$ & $0.11 \pm 0.11$ \\
\hline kurtosis : Sku (no unit) & $6,1 \pm 1.1$ & $4,7 \pm 0.8$ & $5,4 \pm 1.7$ & $3,2 \pm 0.3$ & $2.73 \pm 0.51$ \\
\hline skewness : SsK (no unit) & $1,2 \pm 0.2$ & $0,8 \pm 0.1$ & $0,8 \pm 0.4$ & $0,3 \pm 0.1$ & $-0.07 \pm 0.4$ \\
\hline $\begin{array}{l}\text { developed interfacial } \\
\text { area ratio : Sdr (\%) }\end{array}$ & $79,15 \pm 20.6$ & $79,3 \pm 23.7$ & $139,1 \pm 38.3$ & $351.3 \pm 58.7$ & $3.06 \pm 3.18$ \\
\hline $\begin{array}{l}\text { root mean square } \\
\text { gradient : Sdq (no unit) }\end{array}$ & $1,9 \pm 0.3$ & $1,8 \pm 0.3$ & $2,5 \pm 0.4$ & $4,3 \pm 0.3$ & $0.28 \pm 0.21$ \\
\hline $\begin{array}{c}\text { Polar spectrum } \\
\text { Texture direction : Std }\left(^{\circ}\right)\end{array}$ & & & & & \\
\hline
\end{tabular}

